\title{
Philosophiques
}

\section{"À pas de loup " : incidences narratives de la pensée différée dans les séminaires de Jacques Derrida}

\section{Nicholas Cotton}

Volume 47, numéro 2, automne 2020

Derrida en cours

URI : https://id.erudit.org/iderudit/1075124ar

DOI : https://doi.org/10.7202/1075124ar

Aller au sommaire du numéro

Éditeur(s)

Société de philosophie du Québec

ISSN

0316-2923 (imprimé)

1492-1391 (numérique)

Découvrir la revue

Citer cet article

Cotton, N. (2020). « À pas de loup » : incidences narratives de la pensée différée dans les séminaires de Jacques Derrida. Philosophiques, 47(2), 265-288.

https://doi.org/10.7202/1075124ar
Résumé de l'article

Dans Trois ans avec Derrida, le biographe Benoît Peeters notait que le séminaire de Jacques Derrida était construit " comme un feuilleton théorique qui, par-delà les longueurs et les passages à vide, entretient une sorte de suspense et ménage de réelles surprises d'une séance à l'autre " (Peeters, 2010). Peut-on vraiment rapprocher un séminaire d'une forme romanesque comme le récit différé ? Voilà la question qui nous occupe dans cet article, et nous l'abordons dans une perspective double : à la fois historique et formelle. Nous nous interrogerons ainsi sur ce qu'est un séminaire et comment, dans le cas particulier du séminaire derridien, il fait oeuvre. Le tout nous mène à une réflexion sur l'idée de " pensée différée » à partir du concept central de différance dans l'enseignement derridien. 


\title{
«À pas de loup»: incidences narratives de la pensée différée dans les séminaires de Jacques Derrida
}

\author{
NICHOLAS COTTON \\ Visiting Research Collaborator, Princeton University \\ Professeur de littérature et de français, Collège Édouard-Montpetit
}

\begin{abstract}
RÉSUMÉ - Dans Trois ans avec Derrida, le biographe Benoît Peeters notait que le séminaire de Jacques Derrida était construit «comme un feuilleton théorique qui, par-delà les longueurs et les passages à vide, entretient une sorte de suspense et ménage de réelles surprises d'une séance à l'autre» (Peeters, 2010). Peut-on vraiment rapprocher un séminaire d'une forme romanesque comme le récit différé? Voilà la question qui nous occupe dans cet article, et nous l'abordons dans une perspective double: à la fois historique et formelle. Nous nous interrogerons ainsi sur ce qu'est un séminaire et comment, dans le cas particulier du séminaire derridien, il fait œuvre. Le tout nous mène à une réflexion sur l'idée de «pensée différée » à partir du concept central de différance dans l'enseignement derridien.
\end{abstract}

\begin{abstract}
In Trois ans avec Derrida (Three Years with Derrida), his biographer, Benoît Peeters, notes that Jacques Derrida's seminar was designed "like a theoretical serial which, aside from its slumps and convolutions, nonetheless maintained a kind of suspense and featured genuine cliff-hangers at the end of each session" (Peeters, 2010). Can one truly compare a seminar to a novelistic device like that of deferred narrative? Such is the question to which I devote my attention in this article, a question which is discussed here in a dual - historical and formal - perspective. I thus ask "what is a seminar?" and how, in light of Derrida's seminars in particular, it becomes a work. In closing, I meditate on the notion of "deferred thinking" in Derrida's teaching, using the central concept of différance as my starting point.
\end{abstract}

Pourquoi dirait-on d'un tel séminaire qu'il s'avance à pas de loup? JaCques Derrida, Séminaire La bête et le souverain.

Il va de soi, aujourd'hui, qu'enseignant, je n'enseigne pas la vérité en soi en me transformant en instrument transparent de la pédagogie éternelle, mais que je règle comme je peux un certain nombre de problèmes avec vous et avec moi, et à travers vous et moi avec un certain nombre d'autres instances ici représentées.

JACQUES DERRIDA, La vie la mort.

Dans le livre Trois ans avec Derrida qu'il prépare simultanément à sa biographie du philosophe, Benoît Peeters note systématiquement ses impressions, il recense les entrevues et détaille les obstacles d'écriture, il collige des notes de lecture. Quatre ans seulement après la mort de Jacques Derrida, un premier volume de ses séminaires vient alors de paraître, c'est le Séminaire 
La bête et le souverain. Volume I (2001-2002)1. Lorsque Peeters en entame la lecture, il relève dans son carnet, le 14 novembre 2008, une sorte d'exaspération: "Au début, c'est l'agacement qui domine. Trop de digressions, trop de méandres, un propos éternellement différé. J'ai l'impression que cela traîne, que cela tourne en rond ${ }^{2}$.» Or cet "agacement» des premiers instants s'amenuise cependant que Peeters se laisse gagner par le texte et ses détours:

Mais peu à peu, je me laisse prendre au jeu. Je m'adapte à son rythme. J'accepte le code, les règles, de cette étrange cérémonie que fut le séminaire des dernières années. C'est comme un feuilleton théorique qui, par-delà les longueurs et les passages à vide, entretient une sorte de suspense et ménage de réelles surprises d'une séance à l'autre ${ }^{3}$.

En rapportant ainsi ses impressions et en empruntant à l'analyse romanesque ses notions ( feuilleton", "suspense», etc.), le biographe relève un trait des séminaires qui ne va pas de soi à première vue, lequel mérite néanmoins une attention particulière. Cet article entend explorer cette piste des liens qu'entretient les séminaires de Jacques Derrida avec la forme romanesque, et plus singulièrement avec cette forme qu'on appelle le récit différé. Peut-on vraiment d'abord - et sans préjudice - penser ensemble l'enseignement philosophique et la littérature sérielle? Y a-t-il, ensuite, chez Derrida particulièrement, une manière propre d'aborder l'enseignement en séminaires qui le rapproche des feuilletonistes par exemple? Notre hypothèse est que la forme des séminaires, à supposer que l'on prenne un pas de recul suffisant pour les considérer comme un ensemble, relève effectivement de celle du récit différé ou du moins le rappelle. Pour mener à bien cette démonstration, une perspective double, à la fois historique et formelle, est de mise. Ainsi, nous commencerons par interroger la notion même de séminaire pour ensuite nous consacrer à l'analyse de quelques incidences formelles de cette pratique qui se déploie dans le temps. Cela nous mènera à une réflexion sur la pensée différée et, bien sûr, à la différance.

\section{Les séminaires en France au $x^{\mathrm{e}}$ siècle: Foucault, Lacan et Derrida}

Dans son acception la plus récente et en contexte académique, le mot «séminaire » signifie: "groupe de travail dirigé par un professeur et consacré à un domaine particulier de connaissance ${ }^{4}$. Comme le précise le Petit Robert, il s'agit d'un «Groupe de travail dirigé par un professeur ou un assistant où

1. Jacques Derrida, Séminaire La bête et le souverain. Volume I (2001-2002), Michel Lisse, Marie-Louise Mallet et Ginette Michaud (éds), Paris, Éditions Galilée, 2008.

2. Benoît Peeters, Trois ans avec Derrida. Les carnets d'un biographe, Paris, Flammarion, 2010, p. 129. Nous soulignons.

3. Ibid.

4. Dictionnaire historique de la langue française, Alain Rey (dir.), Paris, Le Robert, 1998, p. 3455. 
les étudiants participent activement ${ }^{5}$ » et, par extension, il peut aussi servir à nommer «l'ensemble des séances de ce groupe ${ }^{6}$ ». Même s'il conserve avec lui un certain rapport de filiation, il faut distinguer cette acception du mot "séminaire" du concept historique - et catholique - de "séminaire ", entendu au sens d'établissement d'enseignement (le grand séminaire où l'on forme les prêtres et le petit séminaire, un «établissement de formation ${ }^{7}$ » où l'enseignement laïque est dispensé à plus large échelle. Étymologiquement, le mot "séminaire" vient du latin classique seminarium (littéralement "pépinière " et, au sens figuré, "source, origine, principe, cause ${ }^{8}$ ») qui tire son origine du génitif semen («semer» en français moderne). Dès 1545, nous renseigne le Dictionnaire historique de la langue française, seminarium renvoie à "un établissement où l'on prépare des jeunes gens à l'état ecclésiastique $^{9}$ ». Selon ses premières occurrences attestées, "séminaire» se comprend ainsi comme ce qui insémine - une idée par exemple - et par conséquent comme ce qui se conçoit dans la durée, dans sa production et donc dans sa potentialité: c'est le «principe vital d'un phénomène ${ }^{10}$ ». Le Séminaire de Jacques Derrida, comme celui de ses contemporains, est peutêtre à rattacher à cette origine analogique, car ceux-ci, de véritables cours magistraux - et rédigés dans le cas de Derrida - étaient construits de manière à faire naître la pensée et à "tourner autour " pour reprendre une figure chère au philosophe. Dans une note de son édition du cours de Derrida, Heidegger: la question de l'Être et l'Histoire (ENS, 1964-1965), Thomas Dutoit rappelle par ailleurs qu'une vieille habitude avait été prise par Derrida et ses étudiants de parler du "Séminaire ", au singulier, terme qu'il préférait à celui de "cours» et qu'il faut en ce sens, c'est le choix éditorial qui a été fait par les éditeurs, distinguer conceptuellement les cours, "orientés par un programme - d'agrégation par exemple ${ }^{11}$ ", des séminaires qui concernent l'enseignement plus libre, notamment à partir de 1984.

Pendant près de quarante-trois ans, Jacques Derrida a enseigné au sein de l'université française, d'abord à la Sorbonne (1960-1964) et à l'École normale supérieure de Paris (1964-1984), puis à l'École des hautes études en sciences sociales (1984-2004). L'enseignement de la philosophie a par conséquent pris une part importante de son temps et constitue un pan méconnu

5. Entrée "Séminaire ", dans Le Nouveau Petit Robert de la langue française, Paris, Le Robert, 2007, p. 2347.

6. Entrée "Séminaire", dans Le Trésor de la langue française, Paul Imbs (dir.), Paris, Éditions du Centre national de la recherche scientifique, 1971, vol. 15, p. 305.

7. Dictionnaire historique de la langue française, p. 3455.

8. Ibid.

9. Ibid.

10. Ibid.

11. Thomas Dutoit, et al., «Introduction générale», dans Jacques Derrida, Heidegger: la question de l'Être et de l'Histoire. Cours de l'ENS-ULM 1964-1965, Thomas Dutoit (éd.), avec le concours de Marguerite Derrida, Paris, Éditions Galilée, 2013, p. 10. 
de son œuvre. Nous pourrions voir dans la popularité de ces séminaires attirant une audience internationale et connaissant un engouement certain depuis le début de leur publication en 2008 un premier point de comparaison avec le récit différé qui a toujours été d'instinct rangé du côté du roman populaire. L'auditorium surpeuplé de l'EHESS où enseigna finalement Derrida à partir de 1984 se substitue à la confidentialité relative des cours donnés à la salle Dussane de l'ENS où se présentaient au plus une cinquantaine d' "initiés" dans les premières années, aux dires d'un journaliste du Nouvel Observateur, Gérard Petitjean, qui a eu l'idée en 1975 de couvrir les cours de cinq "têtes d'affiche ${ }^{12}$ » de l'université française. Peeters, quant à lui, affirme qu'avant cette période déjà plus de cent cinquante auditeurs "s'entass[aient] tant bien que mal ${ }^{13}$ » dans la salle Cavaillès alors que Derrida était assistant de philosophie générale et de logique à la Sorbonne. Les cours de Derrida, autrement dit, étaient dans une certaine mesure de véritables succès, savants comme populaires. À l'instar des grands romans feuilletons du XIX ${ }^{\mathrm{e}}$ siècle ou des séries que proposent actuellement diverses plateformes numériques et télévisuelles, l'enseignement de Derrida suscite tant l'enthousiasme que la fidélité. Ses séminaires, qui attiraient de nombreux étudiants inscrits et auditeurs libres, demeurent bien sûr largement académiques et n'ont que très peu à voir avec les véritables phénomènes de société qu'ont suscité certains feuilletons ou le Game of Thrones de HBO. À son échelle néanmoins, et aidé par une célébrité certaine du philosophe luimême, le séminaire de Derrida fut en son époque l'équivalent universitaire d'un succès commercial. L'exploit n'est toutefois pas le seul à faire école en France au $\mathrm{xx}^{\mathrm{e}}$ siècle, cet enthousiasme académique marque également l'enseignement d'autres grandes figures françaises comme celles de Michel Foucault et de Jacques Lacan.

Selon les éditeurs des Cours que Michel Foucault a donnés au Collège de France, où il fut titulaire d'une chaire sur l'Histoire des systèmes de pensée d'avril 1970 au mois de juin 1984, l'assistance était elle aussi «très nombreuse, composée d'étudiants, d'enseignants, de chercheurs, de curieux, dont beaucoup d'étrangers ${ }^{14}{ }$. Ils précisent même que cette audience «mobilisait deux amphithéâtres ${ }^{15}$ » et que l'affluence était si importante que Foucault, selon l'anecdote, ressentant une telle distance entre lui et cette

12. Gérard Petitjean, «Les grands prêtres de l'université française », Le Nouvel Observateur, 7 avril 1975, p. 53. Voir aussi Dutoit et al., «Introduction générale», p. 12: «En 19641965, à l'ENS, devant un auditoire comportant entre vingt et quarante personnes, élèves de l'ENS, étudiants d'université et auditeurs libres.»

13. Benoît Peeters, Derrida, Paris, Flammarion, 2010.

14. François Ewald et Alessandro Fontana, «Avertissement", dans Michel Foucault, Leçons sur la volonté de savoir. Cours au Collège de France (1970-1971), suivi de Le savoir d'Edipe, F. Ewald, A. Fontana et D. Defert (dir.), Paris, Gallimard et Le Seuil, 2011, p. VIIVIII. Le même «Avertissement» est repris dans tous les volumes.

15. Ibid. 
assistance, modifia l'heure des séances en 1976 - une "méthode sauvage $^{16}$ ", selon les mots du penseur - , les ramenant du soir au matin pour décourager certains étudiants. Gérard Petitjean, que citent les éditeurs de Foucault, précise: "Il y a trois cents places et cinq cents personnes agglutinées, bouchant le moindre espace libre ${ }^{17}$.» Pendant quinze ans, ce sera la même histoire, chaque mercredi de janvier à mars, Foucault se présente en classe moins dans une optique d' " activités d'enseignement ${ }^{18}$ » que pour faire des "comptes rendus publics ${ }^{19}$ » d'un travail en cours, au plus près de l'actualité et de l'histoire, selon une méthode diagonale que décrivent les éditeurs dans leur «Avertissement ${ }^{20}$ ». En outre, le cours s'élaborait "semaine après semaine ${ }^{21}$ » selon la seule double contrainte qu'impose le Collège de France, à savoir celui d'un cours inédit et ouvert à tous. Un autre cas, tout aussi remarquable, est celui des séminaires de Jacques Lacan, qui enseigna quant à lui vingt-six ans, d'abord à l'Hôpital Sainte-Anne (1953-1963), puis à l'École normale supérieure (1964-1969), et enfin à la Faculté de droit du Panthéon (1964-1980)22. Le Séminaire de Lacan est lui aussi abondamment fréquenté, propice à une "catharsis collective» nous dit Élisabeth Roudinesco, l'atmosphère étant celle d'un "banquet socratique» où "chaque auditeur pouvait avoir la certitude que l'orateur s'adressait à lui » ${ }^{23}$. La «cohue ${ }^{24} »$ est, semble-t-il, le nom que donnait Lacan à son propre séminaire.

Rappeler que l'œuvre du psychanalyste était essentiellement oral tient du lieu commun. Lacan refusa longtemps en effet de publier ses cours et lorsqu'il le fait, c'est dans une forme maintes fois retravaillée et excessivement complexe. Les Séminaires contrastent en ce sens avec les Écrits ${ }^{25}$ dont on dit que Lacan les "qualifiait lui-même avec malice [...] d'“illisibles" 26 ". Cela a eu pour résultat de créer une certaine demande pour la transcription des cours eux-mêmes, comme chez Foucault encore une fois et chez Derrida

16. Michel Foucault, "Il faut défendre la société». Cours an Collège de France (1976), F. Ewald, A. Fontana et D. Defert (dir.), Paris, Gallimard et Le Seuil, 1997, p. 4. Voir aussi Ewald et Fontana, "Avertissement", p. VIII, n. 5.

17. Petitjean, «Les grands prêtres de l'université française», p. 54; Ewald et Fontana, «Avertissement», p. VIII.

18. Foucault, «Il faut défendre la société», p. 3.

19. Ibid.

20. Ewald et Fontana, «Avertissement », p. IX.

21. Ibid.

22. Élisabeth Roudinesco, Jacques Lacan. Esquisse d'une vie, histoire d'un système de pensée, Paris, Fayard, 1993, p. 653.

23. Ibid., p. 343.

24. Petitjean, «Les grands prêtres de l'université française », p. 54.

25. Voir Jacques Lacan, Écrits, Paris, Le Seuil, 1999 (2 t.) et Le Séminaire de Jacques Lacan (1953-1979), Jacques-Alain Miller (dir.), Paris, Le Seuil (plusieurs tomes à paraître).

26. Patrick Guyomard, «Lacan, Jacques (1901-1981)», dans Encyclopæedia Universalis, [en ligne: http://www.universalis-edu.com/encyclopedie/jacques-lacan/]; consulté le 14 février 2019. 
plus récemment, où l'édition des séminaires répond en partie à une volonté de contrôler la propagation d'éditions "plus ou moins fiables ${ }^{27}$ ", voire non autorisées. L'École lacanienne de psychanalyse retrace pour sa part l'histoire de cette circulation des «textes» du séminaires:

[D'abord] une sténotypiste assiste Jacques Lacan, dans chacune de ses séances [...]. À l'issue de chaque séance, elle tape à la machine les bandes sténotypées, et ce en double ou triple exemplaires, selon le procédé fameux durant un siècle du «papier carbone». Ces exemplaires sont adressés, à Jacques Lacan [...], le double carbone est envoyé à la trésorière de l’É[cole] F[reudienne de] P[aris] [...]. Jusque vers les années 1970, les séminaires ne circulent pratiquement pas. Le cercle autour de Lacan ne déborde guère celui de ses auditeurs [qui sont par ailleurs nombreux]. [...] À partir des années soixante-dix, [...] Rank Xerox lance la photocopie sèche, rapide et (relativement) économique; et [...] le public autour de Lacan s'agrandit brutalement, [...] de nombreuses versions «transcrites», d'une qualité souvent douteuse, fleurissent jusqu'à la fin. Ce vaste public se tourne volontiers vers les copies des séminaires antérieurs qui — miracle! — surgissent ici et là (presque toujours sous la forme de photocopies des doubles «carbones"), et c'est le boum: elles se multiplient pour répondre aux besoins des "cartels de lecture », qui eux-mêmes les répercutent indéfiniment ${ }^{28}$.

Il y a donc effectivement un authentique emballement autour des séminaires de Foucault et de Lacan et, plus tard, de ceux de Derrida. On se présente à intervalles réguliers, semaine après semaine, pour suivre la pensée en évolution de ces penseurs. On s'échange les transcriptions comme on s'arrachait peut-être certaines livraisons d'un roman publié périodiquement. On remarque en outre de véritables «cartels de lecture» et des réseaux souterrains de distribution de versions non autorisées. Foucault, dans la première leçon du cours "Il faut défendre la société", se montrait particulièrement conscient d'une telle circulation: «il y a toujours des petits micros qui sont là, des appareils d'enregistrement, et comme ça circule ensuite - en certains cas ça reste à l'état de bande, en d'autres cas, ça se retrouve en dactylogramme, quelquefois même, ça se retrouve dans des librairies - alors je me suis dit: ça circulera toujours ${ }^{29}$."

27. À ce sujet et pour tous les détails entourant l'édition des Cours de Michel Foucault, nous renvoyons au très complet: «L'édition des cours et les “pistes" de Michel Foucault. Entretiens avec Mauro Bertani, Alessandro Fontana et Michel Senellart ", entretiens réalisés par Christian Del Vento et Jean-Louis Fournel, Laboratoire italien, nº 7, 2007, p. 173-198. Entre le moment de l'écriture de cet article et celui de sa publication, un collectif, "Free Foucault", s'est même lancé dans la publication des enregistrements des cours dispensés par Michel Foucault au Collège de France: «Free Foucault est une plateforme décentralisée et non-censurable donnant accès à une version restaurée des enregistrements ", [en ligne: https://freefoucault.eth. link/]; consulté le 15 juillet 2020.

28. Voir le site web de l'École lacanienne de psychanalyse, [en ligne: http://www.ecolelacanienne.net/bibliotheque.php? id=13]; consulté le 14 février 2019.

29. Foucault, "Il faut défendre la société», p. 5. 
Contrairement à Lacan qui apportait avec lui «quelques feuillets couverts de notes et de croquis ${ }^{30}$ " et au manuscrit "très inégalement rédigé » duquel Foucault prenait "beaucoup de libertés ${ }^{31}$ ", Derrida rédigeait scrupuleusement tous ses cours. Les quarante-trois ans de séances manuscrites (1960-1969) puis tapuscrites (1969-2003) représentent ainsi quantitativement plus de 14000 pages $^{32}$. Il n'improvisait donc substantiellement qu'à de rares occasions dans le cadre régulier de son séminaire. C'est le cas, par exemple, à la septième séance de son cours Théorie et pratique, publié récemment, où il annonce avoir pris la décision de "transformer quelque chose de [sa] pratique ${ }^{33}$ » en ne se référant plus à un texte préalablement écrit. Mais il abandonne aussi très vite cette nouvelle pratique, séance tenante à vrai dire, proposant qu'une telle improvisation constituait une sorte de fiction, un "dispositif», le réduisant "à la répétition — de [luimême] et du texte commenté ${ }^{34}$ ». Plusieurs séances de séminaire dit «restreint ", cependant, étaient consacrées à l'improvisation et à la discussion, elles servaient justement à briser la formalité d'un enseignement magistral tout en permettant les développements impromptus et les recadrages. Pour y participer, les auditeurs devaient s'impliquer activement par le biais d'exposés et préparer des questions. Ces séances ne font malheureusement pas l'objet d'une transcription systématique dans les volumes publiés.

Dans le séminaire La vie la mort que donne Derrida en 1975-1976, au moment même où Petitjean fait sa tournée des "grands prêtres de l'université française", Derrida s'appuie sur une double lecture de La logique du vivant de François Jacob, et de Sur l'avenir de nos établissements d'enseignement de Friedrich Nietzsche pour rendre apparent le caractère fondamentalement auto-reproducteur d'une institution comme l'université. Dès la première séance de son cours, Derrida postule que «l'université est un système visant par ses programmes, ses contrôles, ses contraintes, à assurer la reproduction de son organisation ${ }^{35}$ ». À la seconde séance, c'est à une analyse de toute la «forme narrative et fictionnelle» des conférences de Nietzsche à laquelle Derrida appelle sans toutefois s'y risquer, faute de temps. L'intrication

30. Roudinesco, Jacques Lacan, p. 343.

31. Bertani et al., "L'édition des cours et les “pistes" de Michel Foucault», p. 185.

32. Geoffrey Bennington et al., "Introduction générale», dans Derrida, La bête et le souverain I, p. 9 (et tous les autres tomes parus chez Galilée).

33. Jacques Derrida, Théorie et pratique. Cours de l'ENS-Ulm 1975-1976 [sic], Alexander García Düttmann (éd.), Paris, Éditions Galilée, 2017, p. 139. Contrairement à ce que propose l'éditeur de ce séminaire et les éditions Galilée qui publient ce volume sous ce titre, nous savons maintenant que ce cours fut donné par Derrida en 1976-1977, suivant le programme d'agrégation de cette année-là dont le thème était «La vie et la mort ». Michael Naas fait cette importante clarification chronologique dans son texte "L'agrégation, le programme: la chance de La vie la mort» publié ici même (p. 315-332).

34. Ibid., p. 141.

35. Jacques Derrida, Séminaire La vie la mort. ENS-Ulm 1975-1976, Pascale-Anne Brault et Peggy Kamuf (éds), Paris, Éditions du Seuil, 2019, p. 26. 
entre séminaire et fiction s'avère donc un thème sur lequel Derrida aurait aimé se pencher plus amplement et il n'est pas trop imprudent d'affirmer que le philosophe a en tête sa propre scène d'enseignement lorsqu'il regrette de ne pas pouvoir étudier chez Nietzsche "ce qui dans des conférences d'un universitaire à des universitaires au sujet de l'université et des lycées, marque déjà une rupture dans l'académisme, dans la scène académique ${ }^{36}$ ».

\section{Séminaire et fiction}

Un séminaire n'est pas une œuvre de fiction. Rien a priori ne devrait être plus évident. Pourtant, si les séminaires de Derrida se présentent comme vrais, ils engagent néanmoins plusieurs procédés narratifs dont le suspense, les rappels et les ralentissements ne sont que les plus apparents. La forme même du séminaire, puisqu'elle s'échelonne dans le temps sans être marquée dès le coup d'envoi par son point d'arrivée, appelle à une analyse comparable à celle d'une narration périodique. Un certain nombre de pratiques hybrides (ni pure vérité ni pure fiction), comme la cure psychanalytique, la lutte américaine ou les campagnes électorales, pour ne donner que ces exemples limites, se rapprochent effectivement de la littérature sérielle. Matthieu Letourneux suggère dans Fictions à la chaîne que ces manifestations - auxquelles nous ajoutons le Séminaire - entraînent des «effets de sérialité » qui «engagent une communication dans laquelle l'œuvre se pense non dans son unicité, mais dans sa relation à un ensemble plus vaste: série de livres, collections, genres, personnages et univers de fiction récurrents ${ }^{37}$ ». Du reste, l'organisation même des séminaires relève incontestablement de la mise en cuvre, sinon de la mise en scène. Si Foucault réfléchissant à sa propre situation pouvait littéralement parler d'un «spectacle ${ }^{38}$ » tout en s'efforçant à un style "limpide et terriblement efficace ${ }^{39}$ ", qui ne faisait aucune place aux effets oratoires, et que Roland Barthes, quant à lui, confiait ne pas supporter la «théâtralisation d'une parole dont [il] se demandai[t] ce qu'elle avait à dire ${ }^{40}$ ", il semble que Derrida ait multiplié les effets de mise en scène. Trois exemples peuvent attester cette pratique.

Le premier est tiré du premier volume du Séminaire La peine de mort (1999-2000). Derrida, dans la langue périphrastique et poétique qu'on lui connaît, se met en scène comme orateur. Il a un souci indéniable pour la narration, comme en témoigne l'incipit de ce volume (séance du 8 décembre 1999):

36. Ibid., p. 66.

37. Matthieu Letourneux, Fictions à la chaîne. Littératures sérielles et culture médiatique, Paris, Seuil, 2017, p. 9.

38. Foucault, "Il faut défendre la société», p. 4.

39. Petitjean, "Les grands prêtres de l'université française », p. 55.

40. Ibid. 
Que répondre à quelqu'un qui viendrait vous dire, à l'aube: «Vous savez, la peine de mort est le propre de l'homme»?

\section{(Long silence)}

Moi, je serais d'abord tenté de lui répondre - trop vite: oui, vous avez raison. À moins que ce ne soit le propre de Dieu - ou que cela ne revienne au même. Puis, résistant à la tentation par la vertu d'une autre tentation - ou en vertu d'une contre-tentation, je serais alors tenté, à la réflexion, de ne pas répondre trop vite et de le laisser attendre — des jours et des nuits. Jusqu'à l'aube.

\section{(Long silence)}

C'est l'aube, maintenant, nous sommes à l'aube. Dans la première lumière de l'aube. Dans la blancheur de l'aube (alba). Avant de commencer, commençons. Nous commencerions.

Nous commencerions par faire semblant de commencer avant le commencement ${ }^{41}$.

Dans cet extrait qui ouvre à proprement parler l'année 1999, Derrida prévoit de longs silences. Ces didascalies, "(Long silence) ", inscrites à même le tapuscrit et oralisées par de longues pauses marquent une tension entre commencement et interruption. Elles théâtralisent le commencement qui est ainsi représenté dans ce passage qui a valeur d'exergue, de commencement avant le commencement. Fond et forme ne font plus qu'un. Mieux encore, c'est la question de la fiction qui est ici rendue perceptible par la mise en scène, celle $\mathrm{du}$ "faire semblant» qui est au cœur de l'argument derridien dans ce volume. L'équivalence entre crime et châtiment qui justifie la peine de mort est une affaire de croyance, veut montrer Derrida dans le sillage de Nietzsche encore une fois: "au fond nous ne croyons pas, nous ne croyons même pas à ce que nous croyons ou disons croire ${ }^{42} "$.

Ces didascalies en rappellent d'autres - deuxième exemple - qui apparaissent quant à elles dans le Séminaire Le parjure et le pardon. Le 4 mars 1998, Derrida entame alors la huitième séance de cet ensemble avec le dialogue suivant:

- Pardon, je m'excuse.

- Mais non, y a pas de mal.

(Répéter) $^{43}$

Dans ce premier volume, Derrida distingue sur le plan conceptuel l'excuse (acquittable) du pardon (ineffaçable) dont il retrace les principales implications sémantiques à partir de plusieurs traditions (abrahamiques et grecque). Lorsqu'il amorce cette séance, Derrida est bien avancé dans cette argumen-

41. Jacques Derrida, Séminaire La peine de mort. Volume I (1999-2000), Geoffrey Bennington, Marc Crépon et Thomas Dutoit (éds), Paris, Éditions Galilée, 2012, p. 23.

42. Ibid., p, 218.

43. Jacques Derrida, Séminaire Le pardon et le parjure. Volume I (1997-1998), Ginette Michaud et Nicholas Cotton (éds), Paris, Éditions du Seuil, 2019, p. 297. 
tation et prévoit, comme en témoigne le fichier informatique de ce cours, déclamer ce dialogue en variant les intonations. Le "(Répéter)» laconique du tapuscrit est ainsi précisé dans ce fichier informatique:

(Mimer et répéter sur tous les tons: un séminaire tout entier aurait pu se limiter à cette théâtralisation des différents tons sur lesquels on peut prononcer ces phrases, en s'épuisant dans la combinatoire, quasiment infinie, des «gestes» de ces phrases, des intonations gestuelles, donc de la "pragmatique » de ce «langage ordinaire», etc. $)^{44}$

Lors de la séance elle-même, Derrida lit effectivement ce dialogue en mimant l'acteur sur la scène. Il ajoute même:

J'imagine un séminaire qui aurait consisté pendant un an à simplement varier [Rires], de façon théâtrale, dramatique, drastique comme on a dit, tous les gestes possibles, la combinatoire de tous ces gestes possibles du "pardon, je m'excuse, mais non, y a pas de mal». Ça aurait suffi, avec de bons acteurs sur cette scène, tout aurait été dit. Comme je ne suis pas un bon acteur, alors je l'explique, mais un bon acteur aurait pu se contenter de répéter ces phrases ${ }^{45}$.

Ici, comme dans la La peine de mort, les didascalies indiquent en un sens une certaine dramaturgie du séminaire qui est déployée par Derrida. S’il rédigeait complètement ses cours, il prévoyait aussi des moments d'improvisation et fixait des périodes pour l'interprétation et le commentaire.

Cette théâtralisation prend une ampleur insoupçonnée dans la deuxième année de ce séminaire - troisième exemple — alors que Derrida propose littéralement de considérer son cours comme une pièce de théâtre. Dès la première séance de cette deuxième année (1998-1999), qui commence par un "(Très lentement) », Derrida se demande: "y a-t-il ou non, devrait-il y avoir ou non une scène, une théâtralité, une mise en scène, voire une obscénité possible de la scène du pardon ${ }^{46}$ ?" Il ajoute ensuite: "Allons donc voir au théâtre, nous y sommes. Allons voir et entendre ${ }^{47}$. » Puis il divise son cours en actes: la première séance devient l' "Acte I, scène 1 " et la sixième l'«Acte VI», tandis que le «rideau» se «lève sur l'acte suivant de notre théâtre historique ${ }^{48}$ à la quatrième séance. Ginette Michaud, d'ailleurs, montre dans sa lecture de ce séminaire à quel point Derrida «tient beaucoup à cette scénographie théâtrale ${ }^{49}{ }^{\prime}$. Ainsi, cette année se présente comme la

44. Ibid., p. 297, note 1.

45. Ibid. Pour l'enregistrement, voir UC Irvine Critical Theory Archive, «Derrida (Jacques) papers ", boîte 110, item MS-C01-A116 «1998 March 4 ».

46. Jacques Derrida, Le parjure et le pardon. Volume II. Séminaire (1998-1999), Ginette Michaud, Nicholas Cotton et Rodrigo Therezo (éds), Paris, Seuil, 2020, p. 29.

47. Ibid.

48. Ibid., "Quatrième séance. Le 20 janvier 1999 ».

49. Ginette Michaud, La vérité à l'épreuve du pardon. Une lecture du séminaire "Le parjure et le pardon" de Jacques Derrida, Montréal, Les Presses de l'Université de Montréal, 2018, p. 59. 
scène d'une intrigue philosophique où tour à tour se présentent quatre personnages de pseudo-fiction sur lesquels jaillit la lumière des projecteurs:

Acte I, scène 1. Des personnages: quatre hommes, quatre noms propres, quatre métonymies ou pseudonymies, quatre figures, quatre "characters", certains de ce temps, et contemporains, certains d'un autre temps, mais peut-être non moins contemporains ni plus anachroniques pour autant: G. Wilhelm Friedrich Hegel, Nelson Mandela, Bill Clinton et Desmond Tutu, autrement dit, le philosophe dialecticien du savoir absolu, les chefs d'État d'Amérique du Nord et d'Afrique du Sud, et le prêtre théologico-politique, représentant éminent de l'Église anglicane.

Le «dispositif» à quatre voix est décrit a posteriori par Derrida dans un autre texte, lors d'une conférence à Rio de Janeiro en 2004 :

Ce séminaire était "composé", voire "dramatisé», mis en scène un peu comme le lieu théâtral d'un tribunal où comparaitraient successivement, en tant que témoins, quatre hommes (et nulle femme), qui étaient aussi des protestants (Hegel, Mandela, Tutu, Clinton). On reconnaîtra ici les traces et justifications de cette mise en scène ${ }^{50}$.

Déjà dans La peine de mort, un an après la deuxième année du séminaire Le parjure et le pardon, Derrida revenait sur ce "dispositif» en réintroduisant au cœur même de sa dramaturgie académique la question de la feinte et de la fiction:

De même que, l'an dernier, nous avions joué sans jouer au théâtre, nous avions feint de jouer à mettre en scène, aussi théâtralement mais aussi peu théâtralement que possible, [à mettre en scène] quatre hommes [...] eh bien cette année, avant de commencer, et parce que la question du théâtre devra retenir notre attention plus encore et autrement que dans la scène sans scène du pardon (l'histoire des rapports entre la peine de mort et le spectacle, la mise en scène, le voyeurisme essentiel qui s'attache à une mise à mort qui doit être publique parce que légale, cette histoire du théâtre de la peine capitale mériterait un séminaire à elle seule et nous y accorderons beaucoup d'intérêt, même si nous ne le faisons jamais assez $[\ldots])^{51}$.

Le dispositif théâtral enjambe donc les deux arcs thématiques que sont Le parjure et le pardon et La peine de mort, se prolongeant cette fois autour de quatre condamnés à mort, à savoir Socrate, Jésus, Hallâj et Jeanne d'Arc.

Encore une fois, Derrida se montre particulièrement sensible aux conditions narratives et à la part de fiction que contient tout acte d'enseignement. Ce jeu de la feinte et de la théâtralisation — un jeu surjoué à bien des égards -, d'une part, sert le propos thématique du séminaire (parjure,

50. Jacques Derrida, «La Conférence de Rio de Janeiro (2004). "Le pardon, la vérité, la réconciliation: quel genre?" ", dans La Solidarité des vivants et le pardon. Conférence et entretiens, Evando Nascimento (éd.), Paris, Hermann, 2016, p. 61, n. 1.

51. Derrida, La peine de mort I, p. 26. 
pardon, peine de mort, souveraineté, etc.) et, d'autre part, déconstruit en acte et sans le nier le statut du professeur et les limites poreuses de la vérité académique. Le philosophe réinscrit à même sa pratique d'enseignement une «fictionnalisation » du séminaire lui-même ou, du moins, du rapport entre enseignement et narration. Il n'est pas rare qu'une partie des séminaires soit de la sorte consacrée, dans des moments fortement autoréférentiels, à la question de la nature du séminaire elle-même. Dans La bête et le souverain où Derrida s'intéresse nommément à la fable «Le loup et l'agneau » de La Fontaine (d'où l'intertexte qui va suivre), ce dernier met clairement son séminaire à distance d'une certaine forme institutionnelle et attendue de l'expérience académique:

En principe, dans la plus noble tradition de l'institution universitaire, un séminaire ne relève pas de la fable. Il n'appartient pas au genre de la fable, $[. .$.$] en$ principe, et suivant sa vocation statutaire, suivant sa loi et le contrat qu'il suppose, le discours enseignant ne doit pas être fabuleux ${ }^{52}$.

La fable serait du côté de la fiction. L'insistance, marquée par la répétition de la locution adverbiale "en principe ", à l'effet qu'un séminaire ne se situe pas du côté de la fiction, laisse entendre que le sien, ce qui a lieu chez lui sous le nom de séminaire, se trouve de l'autre côté, par-delà la limite. À tout le moins, Derrida exhibe tant par le propos que par la forme qu'il remet en question les contours d'une institution qu'il habite et qui l'abrite pourtant. Le philosophe en ajoute même un peu plus, toujours dans La bête et le souverain:

Nous avons commencé ainsi tout en disant qu'aucun séminaire ne devrait commencer ainsi, comme une fable $[. .$.$] comme si ceci restait encore, comme$ séminaire, une fable ou une affabulation [...] compte tenu de ma position accréditée de professeur autorisé à parler ex cathedra pendant des heures, des semaines et des années (accréditée par une convention ou par une fiction dont l'honnêteté reste à prouver, par vous ou par moi $[\ldots])^{53}$.

Enfin, Derrida se montre particulièrement explicite alors qu'il n'hésite plus à parler de "notre histoire ou [de] notre fable intitulée "La bête et le souverain" 54 " à la dixième séance (comme il parlait d'ailleurs, nous l'avons $\mathrm{vu}$, de «notre théâtre historique» quelques années plus tôt). Il devient conséquemment, sinon légitime du moins pertinent, de concevoir le Séminaire comme un récit au long cours duquel se font sentir des effets de sérialisation et des stratégies narratives. Sa forme et son propos nous y autorisent. Quant à cette forme, elle possède en effet certaines caractéristiques qui rappellent les principes du récit différé, que ce soit dans son organisation générale qui relève d'une idée de la continuité narrative (entre ruptures et

52. Derrida, La bête et le souverain I, p. 61. Nous soulignons.

53. Ibid., p. 117. Nous soulignons.

54. Ibid., p. 367. Nous soulignons. 
reprises) ou dans son mode de production - différé dans le temps — qui influence en fait tout le texte. C'est encore ce qu'il nous resterait à démontrer.

\section{Continuité narrative: d'une unité à l'autre}

Pour Matthieu Letourneux, des "phénomènes de sérialisation » sont perceptibles à différents niveaux:

Ils se manifestent d'abord dans la relation des textes aux supports (feuilletons, livraisons, fascicules, livres de poche, etc.). Dans ce cas, chaque format tend à déterminer des modes de consommation (induisant des attentes), des dimensions des textes (et donc des structures narratives, mais aussi des caractéristiques stylistiques et thématiques). En faisant série, il délimite enfin un champ stéréotypique avec des scénarios intertextuels privilégiés.

Sur le plan de la continuité narrative, nous pourrions dire que les séminaires fonctionnent selon une structure sérielle. Nous remarquons en effet qu'au moins quatre échelles correspondent à quatre formes d'unité narrative: l'ensemble du Séminaire, les arcs thématiques ou narratifs, les volumes et les séances. La première de ces unités narratives concerne le Séminaire pris dans sa totalité. Dans La voie aux chapitres, Ugo Dionne propose d'appeler archidispositif ce qui «intervient chaque fois qu'est observable, à un niveau textuel supérieur, une organisation successive ou hiérarchique qui évoque celle de l'opus, sans (nécessairement) présenter son fonctionnement ${ }^{55}$ ». Selon cette typologie, l'archidispositif s'impose comme structure narrative qui fédère un certain nombre de romans (comme dans l'exemple classique de La Comédie humaine). Le Séminaire pris dans sa totalité fonctionne ainsi selon un archidispositif qui se déploie sur quarante-trois ans et conformément à des modalités différentes selon que les cours sont donnés dans une institution ou une autre. À cet effet, il faut rappeler qu'en début de carrière, les thèmes de séminaire étaient imposés à Derrida à raison d'un par année. Dès qu'il commence à enseigner à l'École des hautes études en sciences sociales en 1984, toutefois, il peut choisir lui-même ses projets de recherches.

Premier niveau narratif: l'archidispositif

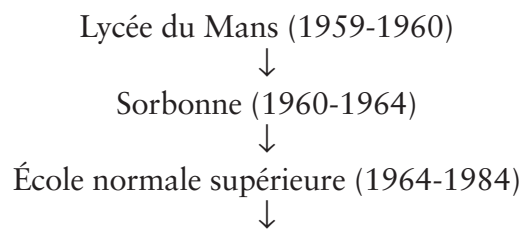

École des hautes études en sciences sociales (1984-2004)

55. Ugo Dionne, La voie aux chapitres. Poétique de la disposition romanesque, Paris, Éditions du Seuil, 2008, p. 21. 
Lorsqu'il est élu directeur d'études à l'École des hautes études en sciences sociales, en décembre 1984, Derrida a tout le loisir de planifier le développement et les prolongements sur plusieurs années de son séminaire. Celles-ci "s'enchaînent de manière explicite, cohérente et saisissante ${ }^{56}$ ". Le projet à grand déploiement intitulé «Les institutions philosophiques» (1984-2004) possède son unité, sa propre cohérence et son autonomie par rapport aux ensembles des autres institutions, qui sont par nature plus hétérogènes, suivant par exemple le programme d'agrégation.

De cette manière, Le parjure et le pardon, La peine de mort et La bête et le souverain sont des sous-ensembles d'un projet lui-même plus englobant ( «Question de responsabilité») où l'on retrouve aussi d'autres sous-ensembles (sous forme de séminaire-année, biennal et parfois triennal). Dès lors, certains arcs narratifs - ce serait une deuxième forme d'unité - dépassent le cadre imposé par l'année académique tout en s'inscrivant dans l'archidispositif.

Deuxième niveau narratif: les arcs

Les institutions philosophiques (EHESS, 1984-2003)

Ensemble 1 (quatre années): Ensemble 2 (trois années):

«Nationalité et nationalisme «Politiques de l'amitié» philosophiques"

Le fantôme de l'autre (1984-1985)

Mythos, logos, topos

(1985-1986)

Le théologico-politique (1986-1987)

Kant, le Juif, l'Allemand

(1987-1988)

(1989-1990)

Manger l'autre II

(1990-1991)
Ensemble 3 (douze années):

"Questions de responsabilité "

Le secret (1991-1992)

Le témoignage I (1992-1993)

Le témoignage II (1993-1994)

Le témoignage III (1994-1995)

Hostilité/hospitalité I (1995-1996)

Hostilité/hospitalité II (1996-1997)

Le parjure et le pardon I (1997-1998)

Le parjure et le pardon II (1998-1999)

La peine de mort I (1999-2000)

La peine de mort II (2000-2001)

La bête et le souverain I (2001-2002)

La bête et le souverain II (2002-2003)

Trois arcs narratifs constituent alors autant d'ensembles à l'intérieur de l'archidispositif: il s'agit des questions de "Nationalité et nationalisme philosophiques» (1984-1988), de «Politiques de l'amitié» (1988-1991) et le plus vaste des trois, celles des "Questions de responsabilité » (1991-2003). On 
remarque alors que la réflexion ne se répète pas, elle se poursuit plutôt d'une année à l'autre. Ainsi, bien qu'une cohérence narrative justifie parfois un changement d'intitulé (on passe par exemple de La peine de mort à La bête et le souverain, ce sera notre troisième niveau narratif), des ponts sont érigés par Derrida qui insiste pour marquer que ses séminaires s'inscrivent dans la continuité. C'est le cas, pour ne donner encore une fois que deux exemples d'une pratique qui est généralisée, lorsqu'il présente le nouvel arc narratif que constitue La bête et le somverain dans l'Annuaire de l'EHESS:

Nous avons poursuivi des recherches qui les années précédentes, autour du problème de la peine de mort, nous avaient conduit à étudier la souveraineté, l'histoire politique et onto-théologique de son concept et de ses figures ${ }^{57}$.

Et encore, ce séminaire était lui-même la suite logique et presque inévitable, si l'on suit Derrida, du précédent (Le parjure et le pardon):

La problématique engagée sous ce titre < «Le parjure et le pardon» > au cours des deux années passées nous a conduits à privilégier cette fois la grande question de la peine de mort. C'était nécessaire au moins dans la mesure où la peine dite capitale met en jeu, dans l'imminence d'une sanction irréversible, avec ce qui paraît tenu pour l'impardonnable, les concepts de souveraineté (de l'État ou du chef d'État - droit de vie et de mort sur le citoyen -), de droit de grâce, etc. ${ }^{58}$

Chaque année d'un sous-ensemble conserve néanmoins une cohésion qui est propre à son volume. Suivant cette idée, c'est la fable "Le loup et l'agneau " de La Fontaine qui organise dans La bête et le souverain, de près ou de loin, la continuité narrative de la première année, alors que c'est le roman Robinson Crusoé de Daniel Defoe et le séminaire de 1929-1930 de Martin Heidegger qui organisent le second volume. Dans les deux cas, c'est plus généralement la question de l'animalité et de la souveraineté qui est le principe unificateur pour les volumes entre eux. La troisième forme d'unité narrative, en ce sens, est contingente ou plutôt conventionnelle: un séminaire se décline sur une année à raison d'environ dix séances, parfois un peu plus, parfois un peu moins, comme nous le montre l'étude des volumes publiés ou en cours de publication.

57. Jacques Derrida, "Questions de responsabilité (IX. La bête et le souverain) ", dans Annuaire de l'EHESS 2001-2002, Paris, Éditions de l'EHESS, 2002, p. 607; [en ligne: https:/ journals.openedition.org/annuaire-ehess/15867]; consulté le 18 février 2019 et cité dans Michel Lisse, Marie-Louise Mallet et Ginette Michaud, "Notes des éditeurs ", dans Derrida, La bête et le souverain I, p. 13.

58. Jacques Derrida, "Questions de responsabilité (VII. La peine de mort)», dans Annuaire de l'EHESS 1999-2000, Paris, Éditions de l'EHESS, 2000, p. 599; cité dans Bennington, Crépon et Dutoit, "Note des éditeurs", p. 15. 
Troisième niveau narratif: le volume

$\begin{array}{llll}\begin{array}{l}\text { Heidegger: la question de l'Être } \\ \text { et l'Histoire }\end{array} & 1964-1965 & \text { ENS-Ulm } & 9 \text { séances } \\ \begin{array}{l}\text { La vie la mort } \\ \text { Théorie et pratique }\end{array} & 1975-1976 & \text { ENS-Ulm } & 14 \text { séances } \\ \begin{array}{l}\text { Le fantôme de l'autre } \\ \text { [Geschlecht III }{ }^{60} \text { ) }\end{array} & 1976-1977^{59} & \text { ENS-Ulm } & 9 \text { séances } \\ \text { Le parjure et le pardon I } & 1984-1985 & \text { EHESS } & 13 \text { séances } \\ \text { Le parjure et le pardon II } & 1998-1999 & \text { EHESS } & 10 \text { séances } \\ \text { La peine de mort I } & 1999-2000 & \text { EHESS } & 8 \text { séances } \\ \text { La peine de mort II } & 2000-2001 & \text { EHESS } & 11 \text { séances } \\ \text { La bête et le souverain I } & 2001-2002 & \text { EHESS } & 13 \text { séances } \\ \text { La bête et le souverain II } & 2002-2003 & \text { EHESS } & 10 \text { séances }\end{array}$

Chaque année correspond donc à un "volume" regroupant chacune des séances données par Derrida entre $17 \mathrm{~h}$ et $19 \mathrm{~h}$ le mercredi, de novembre à mai, et parfois jusqu'en juin. La parution récente du premier volume du séminaire Le parjure et le pardon nous renseigne sur les modalités de ces séances par le biais d'une anecdote qui est loin d'être fortuite. Lors de la toute dernière séance de l'année 1997-1998, dans le cadre du séminaire restreint, Derrida annonce sa retraite, mais il le fait d'une manière qui lui est propre. C'était le 17 juin 1998 :

j'ai longtemps réfléchi à une décision qui m'a paru convenable et qui me ressemble, et j'ai décidé que je ne ferais ni l'un ni l'autre, que je ne partirais pas et je ne resterais pas [Rires]. Et donc, entre les deux, je dis ça pour ceux et celles qui seraient éventuellement intéressés par l'information, j’ai décidé que je continuerais en allégeant les choses, c'est-à-dire en commençant plus tard dans l'année et en arrêtant plus tôt dans l'année. [...] Donc, l'an prochain, je ne commencerai pas début novembre comme d'habitude, mais au début décembre et je ne m'arrêterai pas en juin, mais à Pâques ${ }^{61}$.

À la suite de cette décision, le nombre de séances du séminaire régulier ne change pas vraiment, c'est la période d'enseignement qui est significativement réduite. Par ailleurs, nous savons que Jacques Derrida rédigeait ses

59. Au sujet de ces dates, voir le texte de Michael Naas publié dans ce même numéro, plus loin p. 316-318.

60. Voir Jacques Derrida, Geschlecht III. Sexe, race, nation, humanité, Geoffrey Bennington, Katie Chenoweth et Rodrigo Therezo (éds), Paris, Éditions du Seuil, 2018.

61. Cité dans Ginette Michaud et Nicholas Cotton, «Note des éditeurs", dans Derrida, Le parjure et le pardon I, p. 19. 
séminaires, du moins en partie, "au fur et à mesure de l'année ${ }^{62}$ » au gré de l'avancement des séances. La composition du Séminaire en ce sens coïncide donc avec ce que nous pourrions appeler sa "livraison ", ce qui induit nécessaire des effets à même le texte. Nous pouvons légitimement postuler que les rendez-vous hebdomadaires et le format très strict ( $1 \mathrm{~h} 30$ à $2 \mathrm{~h}$ par séance) auront eu des conséquences sur l'organisation de ces séances et donc sur leur écriture. Les séances inaugurales des séminaires Le parjure et le pardon et La peine de mort, très longues, chevauchent ainsi deux dates dans le calendrier de leur année respective. Ne pouvant pas être lues en une seule livraison, ces séances "débordent " sur la séance suivante où Derrida poursuit le cours de son argumentation après quelques mises au point. Dans les deux cas, ce n'est qu'à la troisième séance que le philosophe peut reprendre l'enchaînement prévu de ses séminaires.

Les séances elles-mêmes forment enfin un quatrième et dernier niveau narratif, minimal, puisqu'elles sont toutes rédigées comme des opus, avec incipit, progression et chute. Il n'est pas rare d'ailleurs que Derrida les publie indépendamment sous forme d'article ou de chapitre de livre ${ }^{63}$. D'un bout à l'autre de cette chaîne d'unités, nous avons ainsi, imbriqués l'un dans l'autre, des séances regroupées en volumes, eux-mêmes regroupés en arcs narratifs thématiques (habituellement un ou deux volumes), qui sont à leur tour assemblés en grands ensembles qui fédèrent un certain nombre de ces thèmes (de deux à sept selon les cas). Du point de vue de la réception, nous nous retrouvons de la sorte devant plusieurs formes d'unité où les parties sont en soi complètes et fragmentaires, effets que multiplie l'édition en cours, ellemême parcellaire et périodique, de chacun des volumes (six volumes publiés depuis 2008 et en principe un par année à partir de 2018). L'approche philosophique derridienne, indissociable des conditions matérielles de sa pro-

62. Bennington et al., "Introduction générale », p. 10.

63. Toute une étude pourrait être menée qui retracerait les publications partielles de certaines séances de séminaire parfois fortement retravaillées, et d'autres fois à peine retouchées dans des articles et des chapitres de livre. Pour s'en tenir ici aux plus importantes, citons les séances reprises dans Jacques Derrida: Acts of Religion, Gil Anidjar (dir.), New York et Londres, Routledge, 2002, dans De l'hospitalité, avec Anne Dufourmantelle, Paris, CalmanLévy, 1997 et dans Politiques de l'amitié, Paris, Éditions Galilée, 1994. Pour la première année du séminaire Le parjure et le pardon seulement, la première séance (12 novembre 1997) a été reprise sous le titre "Le pardon: l'impardonnable et l'imprescriptible", dans le Cahier de L'Herne Derrida, Marie-Louise Mallet et Ginette Michaud (éds), Paris, Éditions de L'Herne, 2004) et fut reprise dans Pardonner. L'impardonnable et l'imprescriptible (Paris, Éditions Galilée, 2012). Des parties de cette même séance furent aussi reprises dans Foi et Savoir (Paris, Éditions du Seuil, 2000). La deuxième séance (26 novembre 1997) a été elle aussi publiée, sous le titre "Qu'est-ce qu'une traduction "relevante" ?", dans Quinzièmes assises de la traduction littéraire (Arles 1998) (Arles, Actes Sud, 1999), puis dans le Cahier de L'Herne. La quatrième séance (14 janvier 1998) a, quant à elle, paru sous le titre «La littérature au secret. Une filiation impossible », dans Donner la mort (Paris, Éditions Galilée, 1999), tandis que les quatre dernières séances (du 25 février au 25 mars 1998) forment «Le ruban de machine à écrire. Limited Ink II », dans Papier Machine (Paris, Éditions Galilée, 2001). 
duction en séminaire, est donc moins progressive que circonvolutive, comme souvent d'ailleurs chez Derrida, elle a pour conséquence d'accroître les effets d'échos, de retours et d'autoréférentialité entre les séances et entre les autres niveaux d'unités. C'est à l'étude de ces traits que nous nous attacherons maintenant.

Pour emprunter deux autres catégories aux théories de la littérature sérielle, il semble que les séminaires, en termes d'archidispositif, tiennent à la fois du cycle et de la série. Dans son livre, Ugo Dionne distinguait effectivement le cycle, dont la prise en considération de l'ensemble comme ensemble est fondamentale, de la série où l'indépendance relative des opus renvoie à une idée commune de lecture sans que la prise en considération de l'ensemble ne soit nécessaire. Ainsi, propose Dionne, dans le cycle, "l'opus y reste toujours autonome, lisible par lui-même; mais il n'acquiert toute sa plénitude sémantique et narrative que lorsqu'on le rapporte au système qui l'inféode ${ }^{64} »$. Le cycle s'élabore par conséquent selon "l'exécution méthodique d'un programme ${ }^{65}$ ", alors que la série est "composé[e] de textes narratifs indépendants, qui renvoient cependant tous à une clé de lecture commune [qui] ne constitue cependant en l'occurrence qu'une proposition, un paradigme que réalise séparément chacun des opus ${ }^{66}{ }^{\prime}$. L'ensemble des séminaires s'apparentent ainsi au cycle parce que les volumes sont autonomes et s'imbriquent dans une structure architectonique plus vaste qui en assure la cohérence, et ce, bien que la prise en compte du cycle ne soit pas toujours nécessaire à la compréhension pleine de chacun des volumes. Geoffrey Bennington, Marc Crépon et Thomas Dutoit, les éditeurs du premier volume de La peine de mort font en effet remarquer dans leur «Note» introductive que:

le lecteur doit avoir à l'esprit dès à présent que les développements, voire les domaines ou les «thèmes» du premier volume [du Séminaire La peine de mort] se distinguent nettement de ceux qui constituent la deuxième année [de ce] séminaire. Si une lecture de l'ensemble peut révéler certains fils conducteurs, elle ne doit pas masquer la façon dont Derrida dissocie les deux années selon les différentes séries ou sous-séries qui les structurent respectivement, sans parler du corpus et des différents champs disciplinaires qui varient d'une année à l'autre ${ }^{67}$.

Il n'y a toutefois pas, comme nous le voyons avec de grands cycles romanesques du genre de la Comédie humaine ou des Rougon-Macquart, une réorganisation a posteriori des volumes qui conservent avec Derrida leur ordre chronologique de rédaction. En ce sens aussi, l'ensemble se rapproche plus de la série où les opus, indépendants, réfèrent à une idée commune de

64. Dionne, La voie aux chapitres, p. 21.

65. Ibid., p. 22.

66. Ibid., p. 21.

67. Bennington, Crépon, et Dutoit, «Note des éditeurs », p. 14. 
lecture (à savoir la reprise d'un topos), comme le sont ici les questions philosophiques de l'auteur et à plus forte raison ses lectures déconstructives. À supposer que nous puissions déjà faire cette expérience qui ne sera possible que dans plusieurs années, lorsque tous les volumes seront publiés, d'une lecture linéaire et chronologique des volumes, cela ne changerait rien à l'affaire, comme en témoigne d'ailleurs la décision éditoriale de publier à rebours les volumes, en partant de celui qui a été donné le plus récemment jusqu'au plus ancien chronologiquement.

En ce qui a trait à la production du séminaire lui-même, plusieurs indices nous permettent de conclure que le cadre périodique des livraisons a eu des incidences textuelles, tantôt mineures, tantôt majeures. Outre le format des séances, tributaire du temps alloué à l'enseignement dans une semaine (deux heures donnent environ trente-quatre pages en moyenne pour les éditions parues chez Galilée et au Seuil), nous avons limité à trois l'analyse de ces incidences textuelles pour les besoins de la démonstration: la fréquence et la temporalité des séances, de même que l'influence du contexte et de l'actualité sur leur création.

La fréquence. Nous pouvons observer que les séances se concentrent généralement du mois de novembre au mois de mai de chaque année (décembre à mars à partir de 1998 pour les raisons que nous évoquions plus haut), ce qui laisse en moyenne une semaine entre chaque livraison et un battement de huit à dix mois entre chaque volume. Le rythme de production est donc régulier, voire inflexible: tout doit être rédigé pour la séance suivante, c'est-à-dire parfois en sept jours. On pourrait croire que Derrida puise dans ses publications le sujet de ses séminaires, mais cela est plutôt rare. Lorsqu'il le fait, il se contente de renvoyer au texte publié en le citant brièvement. Il arrive néanmoins que, de manière plus inusitée, il cite et commente ses propres textes. Pensons notamment à un passage tout à fait singulier du séminaire Le parjure et le pardon tiré d'une séance de séminaire restreint où Derrida relie un texte de Baudelaire à travers la lunette d'une note citée à partir d'un de ses propres textes, Donner le temps:

je ne me relis jamais, mais là, j’ai reçu une réédition de quelque chose [Donner le temps] qui m'a fait penser, qui m'a fait retomber sur un texte [Mon coeur mis à $n u]$ que j'avais cité, pas que j'avais écrit, que j'avais cité, c'est pourquoi je me permets de le reciter ${ }^{68}$.

Puis il enchaîne sur un autre passage concernant plutôt Le Salut par les Juifs de Léon Bloy. Dans les deux cas, c'est l'occasion pour Derrida d'offrir des précisions et de marquer ce qu'il y a d'important dans ces passages. Surtout, c'est l'occasion de faire des rapprochements avec un séminaire qui a eu lieu vingt ans plus tôt, car Donner le temps est lui-même un texte tiré des cinq 
premières séances du séminaire de 1977-197869, appartenant donc à un autre arc narratif. Cela dit, la plupart du temps, c'est plutôt l'inverse qui se produit. Contrairement à Foucault dont les cours «ne redoublent pas les livres publiés, $[\ldots]$ n'en sont pas l'ébauche, $[\ldots]$ ont leur propre statut [et] relèvent d'un régime discursif spécifique dans l'ensemble [de ses] "actes philosophiques"70", le séminaire de Derrida se conçoit comme un laboratoire où les règles de rédaction sont moins strictes et où l'écriture plus exploratoire est presque toujours retravaillée en vue d'une publication, d'un livre ou d'un article, réinvestie à travers le monde par le biais de conférences. À cet égard, la situation des derniers séminaires, à partir de la seconde année du séminaire Le parjure et le pardon, se distingue de celle des premières années par leur caractère largement inédit.

La temporalité. Ce développement de la pensée en plusieurs mois et parfois en plusieurs années imprime nécessairement au texte un certain rapport à la temporalité. Nous retrouvons conséquemment maintes stratégies analeptiques, comme les rappels fréquents, les récapitulations, les mentions de type «l'an dernier nous avons vu X» et «il y a quelques années». Elles foisonnent, et ce, même si Derrida se dit "préfér[er] ne pas consacrer de temps à un retour ou à une récapitulation [et se] contente[r] de rappeler ${ }^{71}$ ». Qui plus est, Derrida use également de stratégies proleptiques du type «retenez X que nous traiterons plus tard » où «plus tard» dans ces cas peut vouloir dire dans un mois, comme lorsqu'il annonce le 11 février 1998 un développement qui ne viendra dans Le parjure et le pardon que le 4 mars $^{72}$, ou plusieurs années plus tard, comme lorsque dans ce même séminaire il annonce à l'avance la thématique de la peine de mort. La mention «la prochaine fois nous parlerons de $\mathrm{X}^{73}$ » clôt quant à elle plusieurs des séances et attise du coup une certaine curiosité. De plus, les auditeurs étaient non seulement nombreux, mais aussi fidèles, ce qui porte Derrida à s'adresser aussi bien à «ceux qui viennent d'arriver» qu'à «ceux qui [lui] font l'amitié et l'honneur de revenir » et qui, étant déjà là il y a quelques années, «s'en souviennent peut-être ${ }^{74}$. Si Derrida fait en outre beaucoup de références aux séminaires des années précédentes et si tout ceci s'apparente certes aux stra-

69. Jacques Derrida, Séminaire «Donner — le temps» [inédit], (1977-1978, ENS-Ulm, Paris). Dans Donner le temps, on peut lire: «le trajet de cet ouvrage correspond fidèlement à celui que j'avais suivi lors des cinq premières séances d'un séminaire donné sous le même titre en 1977-1978 à l'École normale supérieure et l'année suivante à l'Université de Yale ». (Derrida, Jacques, Donner le temps I - La fausse monnaie, Paris, Éditions Galilée, 1991, p. 9.)

70. Ewald et Fontana, "Avertissement », p. IX.

71. Derrida, La bête et le souverain I, p. 142. Nous soulignons. Voir aussi, pour ce volume, p. 106, p. 153 et ailleurs.

72. Derrida, Le parjure et le pardon I, p. 184, p. 188 p. 227 et p. 239.

73. Voir notamment ibid., p. 101 , p. 170 , p. 198 , p. 224 , p. 225 , p. 244 , p. 258 , p. 271 , et p. 323.

74. Ibid., p. 41. 
tégies mémorielles que l'on retrouve dans les séries à grande ampleur, il ne faut pas oublier que cela participe aussi d'un certain déploiement de la pensée propre à la déconstruction. Nous y reviendrons.

Le contexte et l'actualité. L'influence du contexte de production n'est finalement pas négligeable. De multiples imprévus provenant de l'extérieur du séminaire viennent en influencer le cours de telle sorte que nous sommes obligés d'admettre qu'il est à la fois un lieu clos, propre à l'épanouissement d'une pensée autonome par rapport à l'actualité, et à la fois directement tributaire de cette même actualité ${ }^{75}$. Les deux années du séminaire Le parjure et le pardon, où Derrida pense la question du pardon et du parjure de manière généralement théorique dans un premier temps, et à partir de la Commission Vérité et réconciliation et de l'affaire Monica Lewinsky dans un second temps, pourraient de la sorte se penser comme les deux versants d'une réflexion qui se veut à la fois libre et directement en prise sur le présent. De manière plus millimétrique encore, il n'est pas rare que des événements «extérieurs» perturbent le séminaire. Les morts de Jean-Toussaint Desanti et de Pierre Bourdieu ou celle de Maurice Blanchot ${ }^{76}$ qui suscita un vibrant hommage lors de la séance du 26 février 2003 par exemple, viennent influencer le continuum du séminaire. Dans Le parjure et le pardon, le procès pour crime contre l'humanité durant la Seconde guerre mondiale de Maurice Papon, haut fonctionnaire puis ministre français, interpelle Derrida qui en parle d'abord dans une séance du séminaire restreint (le 4 février 1998), puis y revient dans le séminaire courant (le 11 février 1998). Ce procès qui eut lieu en même temps que le séminaire, mais surtout le face-àface auquel il donne lieu entre Papon et Joseph Sitruk, alors Grand Rabbin de France, offre à Derrida une illustration sensible de la différence entre pardon et excuse qu'il essayait d'articuler depuis le début de cette année. Ainsi, le temps investi en séminaire à lire et à commenter des articles de Libération sur le sujet est justifiable «non seulement pour l'intérêt intrinsèque de la chose", comme le dira Derrida, mais aussi nécessaire pour "comprendre la différence au moins conceptuelle entre le pardon et l'excuse $»^{77}$, c'est-à-dire que l'excuse justifie une faute et annule un mal là où le

75. À ce sujet, nous renvoyons à l'article de Maxime Plante dans Cygne noir qui «suggère que la pensée de Jacques Derrida met en échec notre capacité à appliquer une telle distinction [entre nécessité et contingence historique] parce qu'il en va du projet lui-même d'affirmer la nécessité de l'occasionnel au sein de sa pratique textuelle». Voir Maxime Plante, "Jacques Derrida, la sémiologie, le structuralisme: entre nécessité et contingence historique ", Cygne noir, $\mathrm{n}^{\circ} 6$ 2018, [en ligne: http://revuecygnenoir.org/numero/article/plante-derrida-semiologiestructuralisme]; consulté le 4 mars 2019.

76. Voir Derrida, Séminaire La bête et le souverain I, "Quatrième séance ", p. 141-187 pour Desanti et "Cinquième séance», p. 188-222 pour Bourdieu, ainsi que Jacques Derrida, Séminaire La bête et le souverain. Volume II (2002-2003), M. Lisse, M.-L. Mallet et G. Michaud (éds), Paris, Éditions Galilée, 2010, «Septième séance », p. 247-283 pour Blanchot.

77. Derrida, Le parjure et le pardon I, p. 209. 
pardon le garde intact. De même, à la fin de ce volume, Derrida réagit longuement - et quelques jours après seulement - à la demande de pardon qu'avait officiellement formulée, le 24 avril 1998, la chambre des députés allemands pour le bombardement de la ville de Guernica survenu quelque soixante ans plus tôt. Enfin, les auditeurs eux-mêmes influent sur le déroulement des séances qui se modifient en fonction des séances précédentes ou de certaines demandes. Il n'est pas rare en ce sens que Derrida revienne sur une analyse, une démonstration ou un texte étudié. Il change parfois de cap temporairement, prévoit des moments de digression, s'intéresse à des lectures qu'on lui suggère d'une séance à l'autre, comme à tel endroit où Derrida remercie une auditrice «de ne pas avoir oublié et d'avoir localisé » certains passages des Confessions d'Augustin et lui promet de les évoquer "la prochaine fois en commençant ${ }^{78}$. Il arrive même à l'occasion qu'il réponde, comme c'est le cas à la neuvième séance de La bête et le souverain, à «une question très longue et différenciée ${ }^{79}$ » qu'il a reçue par écrit. Les archives du philosophe ${ }^{80}$ regorgent de ces mémos qui témoignent de cette implication épistolaire des auditeurs du Séminaire.

\section{«À pas de loup»}

Si le Séminaire de Jacques Derrida ne peut pas vraiment prétendre à un développement linéaire malgré sa continuité, cela tient aussi et surtout à la nature de la pensée derridienne qui veut s'affranchir d'une démonstration étapiste ou téléologique. Le détour et la marge, tout cela est bien connu, constitueraient la "méthode» privilégiée par Derrida plutôt que l'analyse, la critique et les opérations, c'est ce qu'il suggère au professeur Toshihiko Izutsu dans sa «Lettre à un ami japonais ${ }^{81}$ » au sujet de la déconstruction. Ce serait ce pour quoi Derrida privilégie la périphrase et les circonvolutions. S'il n'est pas faux de le réitérer, ce que nous montrent les séminaires, où l'on peut voir le «professeur Derrida » à l'œuvre, est toutefois infiniment plus nuancé que ce portrait habituellement rapidement brossé du philosophe "déconstructeur » et "iconoclaste». Effectivement, dans le Séminaire, nous sommes en présence d'une pensée de la différance, c'est-à-dire de l'être différent, mais aussi de l'espacement, du retard et donc du mouvement même de différer (pour jouer de l'homonymie avec ce concept de «récit différé» qui nous accompagne depuis le début de ce texte). Pour Charles Ramond dans son Dictionnaire de Derrida: "Le terme de "différance" (avec un a) est [...]

78. Derrida, La bête et le souverain I, p. 139.

79. Ibid., p. 315.

80. Voir notamment «Bibliothèque de Jacques Derrida », Firestone Library, Université de Princeton, Département des livres rares et des collections spéciales, RBD1, boîte B-000634 et boîte B-000634.

81. Jacques Derrida, «Lettre à un ami japonais », dans Psyché II. Inventions de l'autre, Paris, Éditions Galilée, 2003 [1987], p. 9-14. 
l'emblème de la philosophie de Derrida, et sans doute le meilleur exemple de sa manière séduisante et déstabilisatrice, $[. .$.$] c'est le "fait de différer", c'est$ donc, si l'on veut la différence en train de s'établir et non pas établie ${ }^{82}$. I Il s'agit donc littéralement d'une pensée du délai et du détour ou de ce que Derrida nomme souvent la «destinerrance». À l'instar du roman baroque, le Séminaire dans son ensemble se présente comme une pensée construite mais non moins en construction où chaque séance fige un moment particulier, décuple les détours et diffère continuellement le propos.

Prenons une dernière fois l'exemple du premier volume du séminaire La bête et le souverain. Celui-ci annonce et diffère - retarde - l'analyse d'une fable, celle du "Loup et l'agneau». Dans l'extrait que nous avions abordé dès les premières lignes de ce texte, Benoît Peeters décriait l'exaspération causée par "un propos éternellement différé » avant d'y succomber. Cet extrait se poursuit alors que le biographe précise qu'en «[r]etardant indéfiniment le sujet annoncé (le commentaire de la fable "Le Loup et l'Agneau") pour se pencher sur Rousseau, Schmitt, Lacan, Deleuze, Valéry et Celan, le séminaire propose l'aventure d'une méditation à voix haute, le spectacle d'une pensée à ciel ouvert ${ }^{83}{ }^{\prime}$. En fait, l'examen de cette fable qui dévoilerait prétendument le sens du titre du séminaire (La bête et le souverain) est annoncé dès les premières lignes et revient cycliquement de séance en séance, comme chez Marivaux l'annonce de l'histoire de la religieuse dans sa Marianne! Il faut attendre la huitième séance de ce volume pour que Derrida s'avance au-delà des deux premiers vers de la fable de Lafontaine: «La raison du plus fort est toujours la meilleure / Nous l'allons montrer tout à l'heure". C'est sur le deuxième vers, "Nous l'allons montrer tout à l'heure ", qu'insistera le philosophe, car ce dernier met justement l'accent sur la remise à plus tard et touche de ce fait à la structure de tout le Séminaire. En fait, ce vers, "Nous l'allons montrer tout à l'heure ", inséparable des questions qui nous intéressent ici sur la nature et la forme que peut prendre cette pensée différée, revient comme un leitmotiv à chaque séance. Bien que la démonstration donne l'impression - mais l'impression seulement - de se situer dans un avenir incertain et éternellement ajourné, elle est pourtant bel et bien en train de se faire. Dès la première séance, Derrida mentionne: "Imaginez un séminaire [...] qui commencerait ainsi, à pas de loup: "Nous l'allons montrer tout à l'heure." Quoi? Eh bien, "Nous l'allons montrer tout à l'heure" ${ }^{44}$.» Ici, "à pas de loup " caractérise bien ce geste de la philosophie derridienne en général et des séminaires en particulier, sa «méthodologie ", pourrions-nous avancer avec précaution et eu égard à ce qui est dit de la méthodologie dans la "Lettre à un ami japonais». Il faut, autrement dit, entendre l'expression "À pas de loup», comme nous y invite Derrida

82. Charles Ramond, Dictionnaire Derrida, Paris, Ellipses, 2016, p. 66.

83. Peeters, Trois ans avec Derrida, p. 129.

84. Derrida, La bête et le souverain I, p. 26. 
dans son séminaire, à la fois dans son sens littéral: «de façon silencieuse, discrète et inapparente ${ }^{85}$ ", en tournant autour, et de manière plus perverse, c'est-à-dire connotative et homophonique: «il n'y a pas de loup, il y a "pas

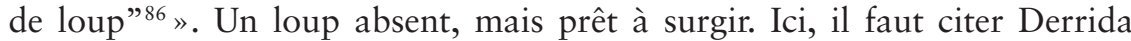
lui-même:

[Le loup] s'annonce, on l'appréhende, on le nomme, on se réfère à lui, on l'appelle même par son nom, on l'imagine ou on projette vers lui une image, un trope, une figure, un mythe, une fable, un phantasme, mais toujours par référence à quelqu'un qui, s'avançant à pas de loup, n'est pas là, pas encore là, quelqu'un qui ne se présente ni ne se représente pas encore; on ne voit même pas sa queue, comme dit encore un autre proverbe.

Tous les séminaires fonctionnent donc ainsi sur le mode du récit différé et de la différance. C'est précisément, nous pouvons maintenant le confirmer, ce qui faisait dire à Peeters dans le passage que nous avons lu en introduction qu'il s'agissait d'une «étrange cérémonie» faite de "trop de digressions » et de «trop de méandres », à savoir d'un "propos éternellement différé ${ }^{87}$ ».

Ce que nous avons étudié n'est vraisemblablement que la pointe d'une recherche formelle et historique qui pourrait être beaucoup plus sérieuse et substantielle, et qui devra porter sur l'ensemble des volumes du Séminaire. Dans les limites que nous nous sommes imposées afin de considérer la forme particulière de l'enseignement en séminaire chez Derrida comme une "narration différée ", nous avons volontairement exclu un certain nombre de pistes qu'il faudrait encore explorer. D'abord, la question du rôle du lecteur des volumes publiés mériterait d'être pensée par rapport aux premiers lecteurs, qui étaient en fait des auditeurs et qui se déplaçaient pour suivre le séminaire. Une autre piste, celle de la différence entre le temps de la production et celui de l'édition, qui survient plusieurs années plus tard (et après la mort de Derrida) pourrait aussi bien mener à une analyse fertile.

Enfin, si l'objet édité et publié chez Galilée ou au Seuil tend à gommer la dimension périodique de l'œuvre dans laquelle nous retrouvons cependant des traces, il semble que l'exercice d'examen de cet objet avec les outils impartis à l'analyse du récit périodique en valait le coup. L'apport est double. Une théorie du récit différée peut, d'une part, nous aider à comprendre les ressorts d'une œuvre complexe comme celle de Jacques Derrida et sa performance. D'autre part, dans le cadre d'une réflexion plus large sur la littérature sérielle elle-même et s'intéressant à des questions de forme et de poétique, un objet limite comme le séminaire (en général) peut trouver sa place, ne serait-ce que dans la remise en question de cette limite.

85. Ibid., p. 23.

86. Ibid., p. 25.

87. Peeters, Trois ans avec Derrida, p. 129. 